\title{
PAH-CHD: transition to adulthood
}

\author{
Andrew Constantine ${ }^{{ }^{*}}$ (D) , Robert M. R. Tulloh ${ }^{2}$, Rebecca Turquet ${ }^{3}$, Konstantinos Dimopoulos ${ }^{1}$ and Shahin Moledina ${ }^{3}$
}

\begin{abstract}
Background: A structured transition provides a framework of care that bridges the gap between paediatric and adult medicine. It is essential for achieving continuity of care and providing support and education around the challenging period of adolescence for young people with pulmonary arterial hypertension associated with congenital heart disease (PAH-CHD).

Presentation: In this review of transition care in $\mathrm{PAH}-\mathrm{CHD}$, we evaluate the evidence supporting a structured programme of transition care and review the current principles and 'best practice' standards for transition in the UK. In the second part of the review, we highlight some important areas of education that are relevant to adolescents with PAH-CHD, including health education, exercise and participation in sports, pregnancy and contraception, employment, and driving.

Conclusions: As the number of young people embarking on transition continues to increase, the challenge is set to continue to improve the quality of care for our patients within the framework of available resources.
\end{abstract}

Keywords: Congenital heart disease, Pulmonary arterial hypertension, Transition, Adolescent medicine

\section{Background}

Pulmonary arterial hypertension $(\mathrm{PAH})$ associated with congenital heart disease (PAH-CHD) is a chronic, incurable condition with a reduced life expectancy and, thus, patients require uninterrupted specialist cardiac care throughout their lives, from childhood to adulthood. Transition describes the structured framework that overarches paediatric and adult care, preparing young people for adulthood and the adult care environment. The process spans over the course of a decade or more starting at the age of 12 years.

All patients with congenital heart disease (CHD) in the UK are expected to undergo a structured transition, run by specialist nurses and overseen by CHD physicians from paediatric and adult services [1]. In principle, therefore, patients with PAH-CHD should also benefit from a CHD transition. However, transition in $\mathrm{PAH}-$ CHD differs to that of other CHD patients as the

\footnotetext{
* Correspondence: constantineah@googlemail.com

${ }^{1}$ Centre for Adult Congenital Heart Disease \& National Centre for Pulmonary Hypertension, Royal Brompton Hospital, London SW3 6NP, UK

Full list of author information is available at the end of the article
}

pulmonary hypertension $(\mathrm{PH})$ service should also be involved, providing PAH-related information, support and education on the disease and medications.

Transition of PAH-CHD patients, is, therefore, a more intensive process, with close coordination between paediatric and adult services and strict monitoring of patients. Indeed, adolescents and young adults with PAHCHD have a reduced life expectancy compared to their peers, may be more symptomatic, and experience a greater impact of their condition on their lifestyle and function. This changes the emphasis and content of information and guidance imparted during the transition period. A structured transition, based on 'best care' principles in both PAH-CHD and CHD, is essential for achieving continuity of care and providing support and education around the challenging period of adolescence.

\section{Importance of a structured transition}

Even though evidence for the effectiveness of structured transition for PAH-CHD patients is lacking, there is widespread recognition that continuous specialist adult CHD care improves outcomes and influences survival 
[2]. Despite this, lapses in follow-up are common and the transition period is a particularly vulnerable time. Kempny et al. retrospectively examined 2 decades of specialist follow-up at a UK specialist CHD centre. Over this period, a quarter of patients failed to attend outpatient clinic appointments and the rate was significantly higher in the young [3]. Non-attendance was predictive of mortality, supporting the need for uninterrupted specialist care. Focusing on the transition period, investigators from the Canadian Adult Congenital Heart (CACH) Network used a lenient criterion of a single specialist outpatient appointment prior to the age of 22 as a marker of successful transition. Yet, only $47 \%$ of patients with CHD met this target [4]. Yeung et al., examined lapses in follow-up in regional CHD clinics in the US. They noted a 3-fold greater need for urgent intervention, such as valve intervention or shunt closure, in the $63 \%$ of patients who had not been seen at regular intervals [5].

In line with these findings, improving transition is a major objective of the UK standards for best practice in CHD [6]. In the UK, the National Institute for Health and Care Excellence (NICE) is an executive public body of the Department of Health, which publishes health guidelines, assessing efficacy and cost-effectiveness. In 2016, NICE released guidelines on transition services [7] laying out the principles behind high quality transition of care.

\section{Transition principles and standards}

The NICE transition guidelines advocate for a personalised programme of support and education, with the aim of gradually increasing knowledge, skills, behaviours and attitudes towards adulthood. Starting from the age of 12 years, the overarching principles of these guidelines are outlined in Table 1.

CHD centres across the UK have agreed on standards for best practice, including transition [6]. The transition components based on these standards are:
Placement of the young person at the centre of the transition process;

Involvement and support of partners, families and carers in discussions around clinical issues, as per the patient's wishes;

Allocation of a Children's Cardiac Transition Nurse (co-ordinator role);

Creation of an individualised, written transition plan;

Review in a specialist multi-disciplinary team transfer clinic by the adult CHD team;

High-quality information transfer between paediatric and adult services (medical records, imaging, individual care plan);

Age and maturity-appropriate information and lifestyle advice;

Opportunity to meet a Practitioner Psychologist on their own, with the availability of psychological support for partners, family or carers;

Formation of a standard operating procedure (SOP) for transition consistent with the standards for $\mathrm{CHD}$ and transition guidelines.

The UK transition process is co-ordinated by a Children's Cardiac Transition Nurse, working in conjunction with a named CHD consultant for each patient. Specialist nurses are likely to have more time to explore issues surrounding the transition period, which can lead to improved care in the teenage population [8]. For young people with $\mathrm{PAH}$ $\mathrm{CHD}$, there must be close collaboration between the $\mathrm{CHD}$ team and $\mathrm{PH}$ services. This requires careful planning, including the formation of 'shared care' clinics, as CHD and $\mathrm{PH}$ services may not be co-located. Other healthcare professionals, including clinical psychologists, palliative care specialists and pharmacists experienced in the care of CHD and PAH patients must be available when needed. Patients' partners, families and carers should be fully supported and there should be recognition of the evolving role of the parent or guardian, to ensure that the patient is able to develop their independence, whilst all parties receive ongoing involvement and support, as required.

Table 1 Overarching principles of transition care as set out in the NICE guidelines [7]

\begin{tabular}{ll}
\hline Principle of transition care & Description \\
\hline Shared organisation of care & $\begin{array}{l}\text { Involving the young person and their carers in the service design, planning, delivery and } \\
\text { evaluation of their own programme of transition } \\
\text { Embracing differences in maturity, cognitive abilities, psychological status, social and } \\
\text { personal circumstances between service users }\end{array}$ \\
Strengths-based support & $\begin{array}{l}\text { Focusing on what is positive and possible for the young person. Identification and } \\
\text { reinforcement of the support available to the young person }\end{array}$ \\
Gradual transfer of responsibility & $\begin{array}{l}\text { Supporting decision-making and developing the ability to direct one's own care over time, } \\
\text { addressing education and employment, community inclusion, health and wellbeing and } \\
\text { independent living } \\
\text { Linking the transition process to a named GP and to colleagues in education }\end{array}$
\end{tabular}


Transfer of care (as opposed to the longer process of transition) from paediatric to adult services usually occurs around 16 years of age. The timing of different components of the transition process should be flexible to allow for differences in the physical and psychological maturity of each patient and their 'preparedness' for transfer. Staged discussions, appropriate to the level of maturity, should occur throughout the transition process, focusing on several areas of teenage and adult life.

There is a significant amount of overlap between CHD and PAH-CHD transition, but the disease severity and medication can translate to a greater impact on lifestyle and function. Clear communication, pitched at a level appropriate for the young person's maturity and understanding, is imperative and allows discussion around important and sometimes difficult topics (e.g. life-long medication with important side-effects, sports, pregnancy etc.).

\section{Areas of focus in PAH-CHD transition}

Education is a vital part of the transition process. A significant proportion of children with PAH-CHD have an associated syndrome and can have learning difficulties. For example, 25\% of patients with Eisenmenger syndrome (ES), currently the largest PAH-CHD subgroup, have Down syndrome. Thus, the level of information communicated, and the degree of involvement of the parent or guardian need to be adjusted to allow the greatest degree of freedom for the young person, while working within a framework of safety and support. After establishing and improving the young person's understanding of their condition, $\mathrm{PAH}$ and the transition process, age-appropriate communication about specific topics relevant to teenagers should be broached in a sensitive and open manner.

\section{Exercise and participation in sports}

Low intensity sporting activities are safe and regular physical activity may optimise healthy development and support long-term cardiovascular health in PAH patients. Individualised activity counselling and exercise prescription allows patients to exercise safely, while limiting the degree of exertion to their symptoms and individual exercise capacity. Very strenuous exercise in the context of PAH may place patients at unnecessary risk. This is exacerbated by cyanosis, as in ES. Thus, participation in competitive sports and endurance activities may carry a prohibitively high risk.

\section{Pregnancy and contraception}

Starting a family is a life goal of many people entering adulthood and having PAH-CHD may not deter some women from becoming pregnant. Unfortunately, patients with PAH are in the highest risk category for adverse outcomes in pregnancy, in whom there is an "extremely high risk of maternal mortality or severe morbidity". The 2018 guidelines for the management of cardiovascular disease during pregnancy advocate discussions about termination in the event of a pregnancy [9]. In contemporary practice, pregnancy-related mortality in the PAHCHD cohort may exceed $20 \%$ and pregnancy is, thus, contraindicated [10]. All people with PAH-CHD choosing to have a baby despite the above information should be under the care of an expert centre for pregnancy and heart disease, with close follow-up for the duration of pregnancy and delivery in a specialist service. Moreover, they should be offered appropriated PAH therapies, whilst interrupting treatments that can be teratogenic.

Due to the prohibitively high risks associated with pregnancy, early discussion about effective contraception is important. The European Society of Cardiology guidelines favour long-acting reversible contraception for patients with heart disease in whom pregnancy is a highrisk endeavour. In patients with PAH-CHD, insertion of intra-uterine devices (IUDs) may trigger a vasovagal response and should be performed in a hospital setting; discomfort and its physiological sequelae may be limited by the new generation of smaller levonorgestrel-based IUDs. Ethinyloestradiol-containing contraceptives carry the greatest thrombotic risk and should be avoided in cyanotic patients and those at risk of thromboembolic phenomena.

\section{Employment and driving}

Canadian data from the $\mathrm{CACH}$ Network suggest that a minority of people with CHD are unable to work for medical reasons, while most individuals are either studying or working full or part-time [4]. Supporting young people to find appropriate employment, for example using specialist career counselling, allows matching of patients' interests and aspirations with their physical abilities. Communication between the transition team and potential employers may help individual applications by accurate risk stratification and identifying issues specific to PAH-CHD. Driving restrictions may impact on career selection and independence. Recent changes in DVLA guidance mean that adults with symptomatic CHD are required to contact the DVLA in order to hold a licence (www.gov.uk/dvla/fitnesstodrive).

\section{Life expectancy and planning for the future}

The acknowledgment and processing of a shortened life span, usually with chronic symptoms, requires a supportive transition environment and a young person who is 'ready' to confront these issues. Average life expectancy in PAH-CHD is disease-specific: In ES, actuarial survival is reduced by approximately 20 years compared 
with healthy individuals, even though many patients die in early life [11]. Idiopathic PAH and PAH in repaired CHD manifesting in childhood carry a much poorer prognosis [12]. With the agreement of parents, information about life expectancy should be imparted and questions answered. The likelihood of future operations or procedures should be discussed to prevent 'surprises' on entering adult CHD care. Difficult conversations about the future can be aided greatly by the presence of a Practitioner Psychologist, especially if already known to the patient. Where life expectancy is significantly reduced or there are symptoms of chronic disease, early referral and close collaboration with the palliative care team is invaluable.

\section{Conclusions}

Transition spans a difficult period in the lives of patients and their family and/or carers. The pillars of transition are effective communication, mutual trust and sustained partnership, from which young people with $\mathrm{PAH}-\mathrm{CHD}$ should derive the tools necessary to tackle the challenges of adolescence and adulthood. Unfortunately, substantial gaps still exist in the provision of transition services, in the UK and abroad. Furthermore, as the number of young people embarking on transition increases, commissioners and service providers will have to balance the rising number of newly transitioning patients with the available resources within the adult $\mathrm{CHD}$ and $\mathrm{PH}$ services, if quality standards are to be met. Continued efforts are needed to set out the best possible design and delivery of transition, through feedback and evaluation of the current transition experience.

\section{Abbreviations}

CACH: Canadian Adult Congenital Heart; CHD: Congenital heart disease; ES: Eisenmenger syndrome; IUD: Intra-uterine device; NICE: National Institute for Health and Care Excellence; PAH: Pulmonary arterial hypertension; $\mathrm{PAH}-$ CHD: Pulmonary arterial hypertension related to congenital heart disease; $\mathrm{PH}$ : Pulmonary hypertension; SOP: Standard operating procedure

\section{Acknowledgements}

Not applicable.

\section{About this supplement}

This article has been published as part of Journal of Congenital Cardiology Volume 4 Supplement 1 2020: Unmet needs in Pulmonary Hypertension associated with Adult Congenital Heart Disease (ACHD-PH). The full contents of the supplement are available at https://jcongenitalcardiology. biomedcentral.com/articles/supplements/volume-4-supplement-1

\section{Authors' contributions}

AC drafted the initial manuscript, which was substantively revised by KD, SM RMRT and RT. All authors read and approved the final manuscript.

\section{Funding}

Medical writing support was provided by nspm Itd, Meggen, Switzerland. The manuscript was funded by a Medical and Educational Goods and Services (MEGS) grant and Actelion Pharmaceuticals UK Limited (who had no influence on manuscript writing).
Availability of data and materials

Not applicable.

Ethics approval and consent to participate

Not applicable.

\section{Consent for publication}

Not applicable.

\section{Competing interests}

Dr. Constantine has received an educational grant from Actelion Pharmaceuticals; Professor Tulloh has received non-financial support from Actelion Pharmaceuticals; Dr. Dimopoulos has received nonfinancial support from Actelion Pharmaceuticals; and has been a consultant to and received grants and personal fees from Actelion Pharmaceuticals, Pfizer, GlaxoSmithKline, and Bayer/MSD. Dr. Moledina has acted as a consultant to Actelion Pharmaceuticals and Bayer.

\section{Author details}

${ }^{1}$ Centre for Adult Congenital Heart Disease \& National Centre for Pulmonary Hypertension, Royal Brompton Hospital, London SW3 6NP, UK. ${ }^{2}$ Bristol Congenital Heart Centre \& South West and South Wales Pulmonary Hypertension Service, Bristol Heart Institute, Bristol, UK. ${ }^{3}$ National Paediatric Pulmonary Hypertension Service, Great Ormond Street Hospital for Children, London, UK

Received: 12 November 2020 Accepted: 12 November 2020 Published: 16 December 2020

\section{References}

1. Dimopoulos K, Favoccia C, Shaughnessy L, et al. Transition to adult care in adolescents with congenital heart disease. Prog Pediatr Cardiol. 2018;51:626.

2. Mylotte $\mathrm{D}$, Pilote $\mathrm{L}$, lonescu-ittu $\mathrm{R}$, et al. Specialized adult congenital heart disease care: the impact of policy on mortality. Circulation. 2015;129:1804-13.

3. Kempny A, Diller GP, Dimopoulos K, et al. Determinants of outpatient clinic attendance amongst adults with congenital heart disease and outcome. Int J Cardiol. 2016;203:245-50.

4. Reid GJ, Irvine MJ, McCrindle BW, et al. Prevalence and correlates of successful transfer from pediatric to adult health care among a cohort of young adults with complex congenital heart defects. Pediatrics. 2004;113: e197-205.

5. Yeung E, Kay J, Roosevelt GE, et al. Lapse of care as a predictor for morbidity in adults with congenital heart disease. Int J Cardiol. 2008;125:62-5.

6. NHS England. Congenital Heart Disease Standards \& Specifications; 2016. p. 147. Available from: https://www.england.nhs.uk/commissioning/wpcontent/uploads/sites/12/2016/03/chd-spec-standards-2016.pdf. Date accessed: 1 June 2020.

7. NICE. Transition from children's to adults' services for young people using health or social care services/Guidance and guidelines; 2018. p. 1-13. Available from: https://www.nice.org.uk/guidance/ng43. Date accessed: 1 June 2020.

8. Habibi $\mathrm{H}$, Emmanuel $\mathrm{Y}$, Chung N. Process of transition for congenital heart patients: preventing loss to follow-up. Clin Nurse Spec. 2017;31:329-34.

9. Regitz-Zagrosek V, Roos-Hesselink JW, Bauersachs J, et al. 2018 ESC guidelines for the management of cardiovascular diseases during pregnancy. Eur Heart J. 2018;39:3165-241.

10. Kiely DG, Condliffe R, Webster V, et al. Improved survival in pregnancy and pulmonary hypertension using a multiprofessional approach. BJOG. 2010; 117:565-74

11. Diller G, Dimopoulos K, Broberg CS, et al. Presentation, survival prospects, and predictors of death in Eisenmenger syndrome: a combined retrospective and case-control study. Eur Heart J. 2006:27:1737-42.

12. Moledina S, Hislop AA, Foster H, et al. Childhood idiopathic pulmonary arterial hypertension: a national cohort study. Heart. 2010;96:1401-6.

\section{Publisher's Note}

Springer Nature remains neutral with regard to jurisdictional claims in published maps and institutional affiliations. 